\title{
Ensemble Kalman filter for model updating - A special issue
}

\author{
Geir Navdal • Remus G. Hanea - Dean S. Oliver • \\ Brice Vallès
}

Published online: 1 March 2011

(C) The Author(s) 2011. This article is published with open access at Springerlink.com

The present issue of Computational Geosciences is a special issue devoted to "Ensemble Kalman filter for model updating". The idea of this issue came after "The 4th International Workshop on Ensemble Kalman Filter for Model Updating", which was held in Bergen in June 2009 (see http://qp.iris.no/enkfseminar). A sequence of international workshops have been held each spring since 2006, in or close to Bergen, Norway, usually attracting more than fifty participants. The workshops are held in an informal atmosphere, giving the participants the possibility to exchange ideas within the

G. Nævdal $(\varangle)$

IRIS, Department of Mathematics,

University of Bergen, Thormøhlensgate 55,

5008 Bergen, Norway

e-mail: Geir.Naevdal@iris.no

R. G. Hanea

TNO Built Environment and Geosciences, Business Unit Geo Energy and Geo Information, TNO,

Delft University of Technology, Princetonlaan 6,

3584 CB Utrecht, The Netherlands

e-mail: remus.hanea@tno.nl

\section{R. G. Hanea}

Department of Applied Mathematics and Department of Geotechnology Section Petroleum Engineering,

Delft University of Technology, Delft, The Netherlands

D. S. Oliver

Uni Research, Center for Integrated Petroleum Research,

P.O. Box 7800, 5007, Bergen, Norway

e-mail: dean.oliver@uni.no

B. Vallès

Department of Physics, Wind Measurement Technologies, UniServices - The University of Auckland, Private Bag 92019, Auckland, New Zealand

e-mail: b.valles@auckland.ac.nz topic. A main objective behind the workshops has been to facilitate communication between users of ensemble Kalman filter (EnKF) in different areas - this has also been a focus of this special issue.

Four of the participants of the 2009 workshop were challenged to prepare a special issue on the "Ensemble Kalman filter for model updating", and this issue is the result achieved in joint efforts with the authors of the papers included.

The EnKF was first introduced in 1994 [1] motivated by applications within oceanographic and atmospheric modeling. The atmospheric and oceanographic communities are clearly dominating in number of publications and applications of the EnKF, but in recent years the EnKF has also been applied for petroleum reservoir models and other subsurface models. Applications in these areas are often challenged with an additional complexity that the effect of unknown model parameters cannot be ignored, which means that these parameters need to be estimated. Although the EnKF is a technique used across several disciplines, most of the papers are published in discipline specific journals. Computational Geosciences, however, has a broad scope, and we therefore hope that the papers published in this issue will reach those interested in EnKF across disciplines.

This special issue contains eight papers. The first three of them focus on localization. The first, P. Sakov and L. Bertino: "Relation between two common localization methods for the EnKF", is discussing localization from a theoretical viewpoint, the second, J. D. Kepert: "Balance-aware covariance localization for atmospheric and oceanic ensemble Kalman filters”, is focusing on problems of particular relevance for atmospheric and oceanic applications, and the third, 
A. Emerick and A. Reynolds: "Combining sensitivities and prior information for covariance localization in the ensemble Kalman filter for petroleum reservoir applications", focuses on issues of particular relevance for reservoir models. The fourth paper, J. Satrom and H. Omre: "Ensemble Kalman filtering with shrinkage regression techniques", suggests methods for improvement in the computation of the Kalman gain matrix to reduce the effects caused by colinearity of ensemble members.

The EnKF is based on an underlying assumption of Gaussianity. For non-linear models, this assumption is not correct. For reservoir models this assumption might also be violated in the geostatistical modeling of the initial permeability and porosity fields. The paper $A$. $S$. Stordal et al.: "Bridging the ensemble Kalman filter and particle filters: The adaptive Gaussian mixture filter" in particular focuses on the effects of the non-linearity, by combining ideas from the EnKF with particle filters through the use of Gaussian mixture models. The paper L. Dovera and E. Della Rossa: "Multimodal ensemble Kalman filtering using Gaussian mixture models”, also applies Gaussian mixture models, but here the focus is on the case for which the Gaussian assumptions is broken in the initial geostatistical description of the reservoir. The paper D. S. Oliver et al.: "Updating Markov chain models using ensemble Kalman filter" considers how to condition discrete variables with a prior described by a Markov chain model to continuous, non-local observations.

The final two papers, L. Peters: "Performance of the ensemble Kalman filter outside of existing wells for a channelized reservoir" and O. Leeuwenburgh et al.: "Assimilation of time lapse seismic data for reservoir history matching with the EnKF" discuss issues concerning the use of the EnKF on reservoir models.

We hope that these papers will stimulate further applications and research of model updating using EnKF, and the editors would like to thank the authors and reviewers for the efforts they have made into preparing this issue.

Open Access This article is distributed under the terms of the Creative Commons Attribution Noncommercial License which permits any noncommercial use, distribution, and reproduction in any medium, provided the original author(s) and source are credited.

\section{Reference}

1. Evensen, G.: Sequential data assimilation with a nonlinear quasi-geostrophic model using Monte Carlo methods to forecast error statistics. J. Geophys. Res. 99(C5), 10,143-10,162 (1994) 